\title{
A TOM DIECK THEOREM FOR STRONG SHAPE THEORY
}

\author{
BERND GÜNTHER
}

\begin{abstract}
We consider an appropriate class of locally finite closed coverings of spaces, for which the strong shape of the elements of the covering and of their intersections determine the strong shape of the whole space. Conclusions concerning shape dimension and spaces having the strong shape of CW-complexes are drawn, and a Leray spectral sequence for strong homology is given.
\end{abstract}

\section{INTRODUCTION}

This paper deals with the reconstruction of global properties of topological spaces from local ones in the following sense: We suppose we are given a covering $\mathscr{A}$ of a space $X$, we know some invariant (e.g. homotopy type, Čech cohomology, etc.) $\lambda(A)$ for the elements $A$ of the covering $\mathscr{A}$ and also for their finite intersections, and we want to find the invariant $\lambda(X)$ of the whole space. In [16, Proposition 4.1] G. Segal has shown that this problem can be solved for $\lambda=$ homotopy type, if $\mathscr{A}$ is a normal open covering, and for $\lambda=$ Čech cohomology, if $\mathscr{A}$ is a locally finite, finite dimensional closed covering of a paracompact space. In [6] tom Dieck explored the normal open case further and concluded, that a map $f: X \rightarrow Y$ is a homotopy equivalence, if there exists a normal open covering $\mathscr{U}$ of $Y$, such that for every finite collection of sets $U_{0}, \ldots, U_{n} \in \mathscr{U}$ the restricted map $f: f^{-1}\left(\bigcap_{k=0}^{n} U_{k}\right) \rightarrow \bigcap_{k=0}^{n} U_{k}$ is a homotopy equivalence. Dydak and Nowak proved in [7], that this theorem holds for locally finite closed coverings of paracompact spaces and for homotopy equivalences replaced by strong shape equivalences. This naturally leads to the question, how Segal's techniques work in the strong shape category. Also we want to examine suitable conditions for coverings in order to ensure Dydak's and Nowak's result beyond the class of paracompact spaces.

An appropriate class of "admissible" coverings will be presented in $\S 2$. It turns out that our problem is related to expansion properties of coverings: In [10] Katětov showed that every locally finite covering of a collectively normal, countably paracompact space has a locally finite open expansion. Krajewski and

Received by the editors May 20, 1991.

1991 Mathematics Subject Classification. Primary 54C56, 54D20, 55P55.

Key words and phrases. Locally finite closed coverings, expansion of coverings, strong shape, strong homology, shape dimension, tom Dieck Theorem.

This article was written during a visit at the University of Washington, Seattle, sponsored by the DFG. The author enjoyed many inspiring discussions with Jack Segal, Ernest Michael, Stanislaw Spież and Roman Pol. 
Smith $[12,17]$ examined the class of all topological spaces sharing this property, and called them expandable spaces. We will take the converse point of view and give a sufficient condition for coverings, such that the expansion property holds in all topological spaces.

In $\S 3$ we give applications concerning shape dimension, spaces of trivial shape, spaces having the strong shape of a $\mathrm{CW}$-space and spectral sequences for strong homology or Čech cohomology.

For a treatment of strong shape theory of arbitrary topological spaces we refer to $[5,7,13]$. The only knowledge required for our present purpose is a characterization of continuous mappings, which are invertible in the strong shape category, to be found in [7, Definition 1.1]: A map $f: X \rightarrow Y$ is a strong shape equivalence if and only if it has the following two properties:

(i) Any map from $X$ into an ANR-space can be factored over $f$ up to homotopy and

(ii) if two maps $g_{0}, g_{1}: Y \rightarrow P$ into an ANR-space $P$ and a connecting homotopy $H: g_{0} f \simeq g_{1} f$ are prescribed, then there exists a homotopy $G: g_{0} \simeq g_{1}$ with $G(f \times$ id $) \simeq H$ relative $X \times \dot{I}$.

\section{ADMISSIBLE COVERINGS}

We will have to assume that our covering is composed of normally embedded sets. Normally embedded sets are characterized as follows:

Lemma 1. A subspace $A$ of a topological space $X$ is normally embedded if and only if it has the following property: For any two maps $f: A \rightarrow P, \varepsilon: A \rightarrow$ ] $0, \infty[$, where $P$ is an ANR-space equipped with a specific metric $d$ generating its topology, there exist a normal neighborhood $W$ of $A$ in $X$ and a map $g: W \rightarrow P$, such that for all $x \in A$ the inequality $d(f(x), g(x))<\varepsilon(x)$ holds. If $P$ is an $A R$ we can choose $W=X$.

We recall that a neighborhood $W$ of $A$ in $X$ is called normal, if its complement can be separated from $A$ by an Urysohn function.

Proof. We assume that $A$ is normally embedded in $X$ and consider the ANRspace $Q:=P \times] 0, \infty[$, the covering $\mathscr{U}$ of $Q$ formed by the open sets $U(x, t)$ $:=\left\{(y, s) \in Q\left|d(x, y)<\frac{1}{4} t,\right| s-t \mid<\frac{1}{2} t\right\}$ and the map $F: A \rightarrow Q$ with components $F=(f, \varepsilon)$. By [9, Theorem 2.3.a] there is a normal neighborhood $W$ of $A$ in $X$ and a map $G=(g, \delta): W \rightarrow Q$, whose restriction to $A$ is $\mathscr{U}$-near to $F$. Then $g$ satisfies $d(f(x), g(x))<\varepsilon(x)$ for all $x \in A$. If $P$ is an AR then we can find a contracting homotopy $H: P \times I \rightarrow P, \quad H_{0} \equiv p_{0} \in P$ and $H_{1}=$ id. We take an Urysohn function $\varphi: X \rightarrow I$ with $\varphi \equiv 1$ on $A$ and $\varphi \equiv 0$ on $X \backslash W$ and replace $g$ by the function $g^{\prime}: X \rightarrow P$ defined as follows:

$$
g^{\prime}(x):= \begin{cases}H(g(x), 2 \varphi(x)-1), & \varphi(x) \geq 1 / 2, \\ p_{0}, & \varphi(x) \leq 1 / 2 .\end{cases}
$$

Now let us suppose that $A$ has the property formulated in our lemma. To show that $A$ is normally embedded in $X$ we want to apply [9, Theorem 2.3.a] and consider an open covering $\mathscr{U}$ of an ANR-space $P$ and a map $f: A \rightarrow P$. For each $p \in P$ we set $\delta^{\prime}(p)$ equal to the supremum of all $\delta^{\prime}>0$, such that 
the open ball around $p$ of radius $\delta^{\prime}$ is contained in a member of $\mathscr{U}$. Then $\delta^{\prime}$ is a strictly positive, lower semicontinuous function on the metrizable space $P$, and by a Theorem of Dowker [8, Problem 5.5.20.a, p. 347] there is a strictly positive, continuous function $\delta$ on $\mathrm{P}$ with $\delta \leq \delta^{\prime}$. Our lemma ensures the existence of a normal neighborhood $W$ of $A$ in $X$ and a map $g: W \rightarrow P$ with $d(f(x), g(x))<\delta(f(x))$ for all $x \in A$, and this means that $f$ and $g$ are $\mathscr{U}$-near on $A$.

Let $X$ be a topological space and $\mathscr{A}=\left\{A_{\alpha} \mid \alpha \in M\right\}$ an arbitrary covering of $X$. We recall that the nerve $\mathscr{N}(\mathscr{A})$ of $\mathscr{A}$ is the simplicial complex, whose vertices are the indices $\alpha \in M$, and whose simplexes are those finite collections of indices $\varnothing \neq \sigma \subseteq M$ with $A_{\sigma}:=\bigcap\left\{A_{\alpha} \mid \alpha \in \sigma\right\} \neq \varnothing$. The geometric realization of $\mathscr{N}(\mathscr{A})$ with the CW topology is denoted by $N(\mathscr{A})$, and by $X \overline{\times} N(\mathscr{A})$ we mean the cartesian product of these two factors carrying the weak topology determined by the subspaces of the form $X \times K$ with $K$ running over all compact subspaces of $N(\mathscr{A}) .|\sigma| \subseteq N(\mathscr{A})$ is the closed geometric simplex spanned by the vertices $\alpha \in \sigma$.

Definition 1. $X^{\mathscr{A}} \subseteq X \overline{\times} N(\mathscr{A})$ is the subspace obtained as the union of the sets $A_{\sigma} \times|\sigma|$ with $\sigma$ ranging over the simplexes of $\mathscr{N}(\mathscr{A})$.

Let $\widetilde{N}$ be the full complex containing $\mathscr{N}(\mathscr{A})$, i.e. both complexes have the same set of vertices and any finite, nonvoid collection of vertices determines a simplex of $\widetilde{N} . \widetilde{N}$ is the corresponding geometric realization; we observe that $\widetilde{N}$ is contractible.

Proposition 1. If every finite intersection of elements of $\mathscr{A}$ is normally embedded in $X$, then $X^{\mathscr{A}}$ is normally embedded in $X \overline{\times} \widetilde{N}$.

Proof. We want to apply Lemma 1 and consider a map $f: X^{\mathscr{A}} \rightarrow P$, a metric $d$ on $P$ generating its topology and a map $\left.\varepsilon: X^{\bowtie} \rightarrow\right] 0, \infty[$; we may suppose that the metric $d$ is bounded. $P$ can be isometrically embedded in a normed vector space, such that $P$ is closed in its convex hull $K$. Then there is an open neighborhood $U$ of $P$ in $K$ and a retraction map $r: U \rightarrow P$. Let $V$ be a second open neighborhood of $P$ in $K$ whose closure is contained in $U$. We claim:

(iii) There is a strictly posivite, real valued, continuous function $\delta$ on $X^{\mathscr{A}}$, such that for each $z \in X^{\mathscr{A}}$ the open ball in $K$ around $f(z)$ with radius

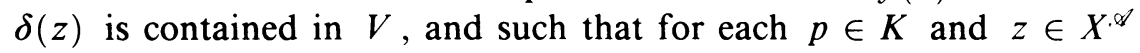
the relation $\|f(z)-p\|<\delta(z) \Rightarrow\|f(z)-r(p)\|<\varepsilon(z)$ holds.

(iv) We consider a normally embedded subspace $B$ of a space $Y$ and maps $\varphi: Y \times S^{n-1} \rightarrow K, \psi: B \times E^{n} \rightarrow K$ and $\left.\delta_{1}, \delta_{2}: B \times E^{n} \rightarrow\right] 0, \infty[$ with $\delta_{1}<\delta_{2}$ on $B \times E^{n}$ and $\|\varphi(b, t)-\psi(b, t)\|<\delta_{1}(b, t)$ for all $(b, t) \in B \times S^{n-1}$. Then there is $\omega: Y \times E^{n} \rightarrow K$ with $\omega=\varphi$ on $Y \times S^{n-1}$ and $\|\omega(b, t)-\psi(b, t)\|<\delta_{2}(b, t)$ for all $(b, t) \in B \times E^{n}$.

We suppose for the moment that these two claims are true and take a strictly increasing sequence of strictly positive numbers $\rho_{n}$ converging to 1 . For any finite, nonempty subset $\mu \subseteq M$ containing $n$ elements we denote by $|\mu| \subseteq \widetilde{N}$ the simplex spanned by the elements of $\mu$. Claim (iv) allows us to construct a 
family of maps $\bar{g}_{\mu}: X \times|\mu| \rightarrow K$ by induction on $n$, subject to the following two conditions:

(v) For $\nu \subseteq \mu$ the map $\bar{g}_{\nu}$ is the restriction of $\bar{g}_{\mu}$.

(vi) If $\sigma$ is an $n$-simplex of $\mathscr{N}(\mathscr{A})$, then $\bar{g}_{\sigma}$ satisfies the inequality

$$
\left\|\bar{g}_{\sigma}(x, t)-f(x, t)\right\|<\rho_{n} \delta(x, t)
$$

for all $x \in A_{\sigma}$ and $t \in|\sigma|$.

The collection of all these maps determines a map $\bar{g}: X \overline{\times} \widetilde{N} \rightarrow K$ with $\|\bar{g}(x, t)-f(x, t)\|<\delta(x, t)$ for all $(x, t) \in X^{\mathscr{A}}$. Claim (iii) implies $\bar{g}\left(X^{\mathscr{}}\right)$ $\subseteq V$, so that $W:=\bar{g}^{-1}(U)$ is a normal neighborhood of $X^{\mathscr{A}}$ in $X \overline{\times} \widetilde{N}$, and by (iii) the map $g:=r \bar{g}: W \rightarrow P$ satisfies $\|f(x, t)-g(x, t)\|<\varepsilon(x, t)$ for all $(x, t) \in X^{\mathscr{A}}$. Now Lemma 1 implies that $X^{\mathscr{A}}$ is normally embedded in $X \overline{\times} \widetilde{N}$.

To prove (iii) we introduce a continuous pseudometric $\rho$ on $X^{\mathscr{A}}$ by

$$
\rho\left(z_{1}, z_{2}\right):=\left\|f\left(z_{1}\right)-f\left(z_{2}\right)\right\|+\left|\varepsilon\left(z_{1}\right)-\varepsilon\left(z_{2}\right)\right| .
$$

If $Z$ denotes the metric space obtained from $X^{\mathscr{A}}$ by identifying points of $\rho$-distance 0 , then $f$ and $\varepsilon$ may be considered as continuous functions on $Z$. For each point $z$ of this metric space we denote by $\delta^{\prime}(z)$ the supremum of all positive real numbers $\delta^{\prime}$, such that the open ball in $K$ around $f(z)$ is contained in $V$, and such that for all $p \in V$ the relation $\|f(z)-p\|<\delta^{\prime} \Rightarrow$ $\|f(z)-r(p)\|<\varepsilon(z)$ holds. Then $\delta^{\prime}$ is a lower semicontinuous function on $Z$ and by Dowker's Theorem there is a continuous function $\delta$ on $Z$ with $0<\delta<\delta^{\prime}$. This function has the required property.

To prove (iv) we set $\left.\eta:=\frac{1}{4}\left(\delta_{2}-\delta_{1}\right): B \times E^{n} \rightarrow\right] 0, \infty[$ and take a map $\Psi: Y \times E^{n} \rightarrow K$ with $\|\Psi-\psi\|<\eta$ on $B \times E^{n}$ applying Lemma $1 ; \Psi$ exists because $B \times E^{n}$ is normally embedded in $Y \times E^{n}$. For $b \in B$ we define $\left.\vartheta^{\prime}(b) \in\right] 0,1[$ to be the supremum of all numbers $\left.\vartheta \in] 0,1 / 2\right]$, such that $\|t\|>$ $1-\vartheta$ implies $\|\varphi(b, t /\|t\|)-\Psi(b, t)\|<\delta_{1}(b, t)+\eta(b, t)$. The function $\vartheta^{\prime}$ : $B \rightarrow$ ]0, 1[ is lower semicontinuous with respect to the continuous pseudometric $\rho$ on $B$ given by $\rho\left(b_{1}, b_{2}\right):=\left\|\varphi\left(b_{1}\right)-\varphi\left(b_{2}\right)\right\|+\sup _{t}\left\|\Psi\left(b_{1}, t\right)-\Psi\left(b_{2}, t\right)\right\|$, and by Dowker's Theorem there is a continuous map $\left.\vartheta^{\prime \prime}: B \rightarrow\right] 0,1[$ with $\vartheta^{\prime \prime}<\vartheta^{\prime}$. Applying Lemma 1 we find a continuous map $\left.\vartheta: Y \rightarrow\right] 0$, 1[ with $\left|\vartheta-\frac{1}{2} \vartheta^{\prime \prime}\right|<\frac{1}{2} \vartheta^{\prime \prime}$ on $B$, especially $\vartheta<\vartheta^{\prime \prime}$. Now we can define $\omega: Y \times E^{n} \rightarrow K$ by

$$
\omega(y, t):=\left\{\begin{array}{l}
\Psi(y, t), \quad\|t\| \leq 1-\vartheta(y), \\
\vartheta(y)^{-1}\{(1-\|t\|) \Psi(y, t)+(\|t\|-1+\vartheta(y)) \varphi(y, t /\|t\|)\}, \\
1-\vartheta(y) \leq\|t\|,
\end{array}\right.
$$

and this map satisfies all requirements.

Lemma 2. We consider two closed subsets $A$ and $B$ of a topological space $X$, whose union is normally embedded in $X$, and a normal neighborhood $U$ of $A \cap B$. Then there exist normal neighborhoods $V_{A}$ and $V_{B}$ of $A$ respectively $B$ in $X$ with $V_{A} \cap V_{B} \subseteq U$. 
Proof. Let $\varphi: X \rightarrow I$ be an Urysohn function with $\varphi \equiv 1$ on $A \cap B$ and $\varphi \equiv 0$ on $X \backslash U$. We define $\psi^{\prime}: A \cup B \rightarrow I$ by

$$
\psi^{\prime}(x):= \begin{cases}\frac{1}{2} \varphi(x), & x \in A, \\ 1-\frac{1}{2} \varphi(x), & x \in B .\end{cases}
$$

Since $A \cup B$ is normally embedded in $X$ Lemma 1 provides us with a map $\psi: X \rightarrow I$, such that for all $x \in A \cup B$ the inequality $\left|\psi(x)-\psi^{\prime}(x)\right|<1 / 8$ holds. We set

$$
\begin{aligned}
& V_{A}:=\{x \mid \psi(x)<1 / 2\} \cup\{x \mid \varphi(x)>0\}, \\
& V_{B}:=\{x \mid \psi(x)>1 / 2\} \cup\{x \mid \varphi(x)>0\},
\end{aligned}
$$

then evidently we have $V_{A} \cap V_{B} \subseteq U$. To show that $V_{A}$ is a normal neighborhood of $A$ we consider the map $\vartheta_{A}: X \rightarrow I$,

$$
\vartheta_{A}(x):=\min \left\{1,4 \max \left(\frac{1}{2}-\psi(x), \varphi(x)\right)\right\} .
$$

This implies $\vartheta_{A}(x)>0 \Leftrightarrow x \in V_{A}$, and we claim $x \in A \Rightarrow \vartheta_{A}(x)=1$. This is clear for $\varphi(x) \geq 1 / 4$, and for $\varphi(x)<1 / 4$ we have $\psi^{\prime}(x)<1 / 8$ and therefore $\psi(x)<1 / 4 \Rightarrow \vartheta_{A}(x)=1$. The neighborhood $V_{B}$ of $B$ is treated similarly.

Definition 2. A family of subsets $\mathscr{A}=\left\{A_{\alpha} \mid \alpha \in M\right\}$ of a topological space $X$ will be called admissible if

(vii) every element of $\mathscr{A}$ is closed,

(viii) every finite union of finite intersections of elements of $\mathscr{A}$ is normally embedded in $X$ and

(ix) there is a normal open covering $\mathscr{U}$ of $X$ every member of which intersects only finitely many members of $\mathscr{A}$.

Remark 1. (a) If $X$ is paracompact, then admissible families coincide with locally finite closed families.

(b) By definition a family of subsets $\mathscr{A}$ of $X$ is locally finite if there is an open covering $\mathscr{U}$ of $X$, whose members intersect only finitely many members of $\mathscr{A}$. However, if $X$ is not paracompact this definition is not too useful; it improves if $\mathscr{U}$ is assumed to be normal.

(c) We observe that the union of two normally embedded closed subspaces need not be normally embedded; for instance a two point subspace of a nonHausdorff $T_{1}$-space is normally embedded if and only if the two points can be seperated by an Urysohn function. If the space $X$ is normal, then the union of two closed subspaces $A$ and $B$ is normally embedded if $A, B$ and $A \cap B$ are normally embedded.

Proposition 2. Let $\mathscr{A}=\left\{A_{\alpha} \mid \alpha \in M\right\}$ be an admissible family of subsets of a space $X$, and suppose that for any nonempty finite subset of indices $\mu \subseteq M$ we are given a normal neighborhood $U_{\mu}$ of $A_{\mu}:=\bigcap\left\{A_{\alpha} \mid \alpha \in \mu\right\}$. Then we can assign a normal neighborhood $V_{\alpha}$ of $A_{\alpha}$ in $X$ to each $\alpha \in M$, such that for every nonvoid finite $\mu \subseteq M$ the intersection $\bigcap\left\{V_{\alpha} \mid \alpha \in \mu\right\}$ is contained in $U_{\mu}$. 
Remark 2. Proposition 2 implies in particular that any admissible family of pairwise disjoint sets may be enlarged to a pairwise disjoint open family. This is a remnant of collectionwise normality valid for arbitrary spaces.

Proof. At first we deal with the special case of a finite family $\mathscr{A}=\left\{A_{k} \mid 1 \leq k\right.$ $\leq n\}$. For $n=1$ there is nothing to prove, so let us consider the case $n+1$. We apply the induction hypothesis twice: On the one hand to the sets $A_{k}$ for $1 \leq k \leq n$ and the neighborhoods $U_{\mu}$ for $\varnothing \neq \mu \subseteq\{1, \ldots, n\}$ and on the other hand to the sets $A_{k} \cap A_{n+1}$ and the neighborhoods $U_{\mu \cup\{n+1\}}$ with $k$ and $\mu$ as above. We get normal neighborhoods $V_{k}^{1}$ of $A_{k}$ and $V_{k}^{2}$ of $A_{k} \cap$ $A_{n+1}$ with $\bigcap\left\{V_{k}^{1} \mid k \in \mu\right\} \subseteq U_{\mu}$ and $\bigcap\left\{V_{k}^{2} \mid k \in \mu\right\} \subseteq U_{\mu \cup\{n+1\}}$. Lemma 2 provides us with normal neighborhoods $V_{k}$ of $A_{k}$ and $V_{n+1}$ of $A_{n+1}$ with $V_{k} \cap V_{n+1} \subseteq V_{k}^{2}$, and we may assume $V_{k} \subseteq V_{k}^{1}$ and $V_{n+1} \subseteq U_{n+1}$. This ensures $\bigcap\left\{V_{k} \mid k \in \mu\right\} \subseteq U_{\mu}$ for all nonvoid $\mu \subseteq\{1, \ldots, n+1\}$.

Now we turn to the infinite case. By condition (ix) there is a normal covering $\mathscr{W}$ of $X$, each of whose members intersects only finitely many members of $\mathscr{A}$. Let $\mathscr{W}^{\prime}$ be a normal star refinement of $\mathscr{W}$ and $\left\{\varphi_{l}\right\}$ a locally finite partition of unity on $X$ subordinated to $\mathscr{W}^{\prime}$. We define a continuous pseudometric $d$ on $X$ (not necessarily generating the topology) by

$$
d(x, y):=\frac{1}{2} \sum_{l}\left|\varphi_{l}(x)-\varphi_{l}(y)\right| ;
$$

then any two points with distance less than 1 are $\mathscr{W}^{\prime}$-near. By $W_{x}$ we denote the open ball around $x$ of radius 1 with respect to $d$, and by $W_{x}^{\prime}$ the open ball with radius $1 / 2$. We observe that every $W_{x}$ meets only finitely many members of $\mathscr{A}$.

We already know that to any index $\alpha \in M$ and finite subset $\mu \subseteq M$ containing $\alpha$ we can assign a normal neighborhood $V_{\alpha}^{\mu}$ of $A_{\alpha}$ with $\bigcap\left\{V_{\alpha}^{\mu} \mid \alpha \in \nu\right\} \subseteq$ $U_{\nu}$ for every nonvoid $\nu \subseteq \mu$, and in addition we may assume $V_{\alpha}^{\mu} \subseteq V_{\alpha}^{\nu}$ for $\nu \subseteq \mu$. We define sets $V_{\alpha} \subseteq X$ by

$$
x \in V_{\alpha}: \Leftrightarrow d\left(x, A_{\alpha}\right)<1 / 2 \text { and } x \in V_{\alpha}^{\mu} \text { with } \mu:=\left\{\beta \mid W_{x}^{\prime} \cap A_{\beta} \neq \varnothing\right\} .
$$

We observe that $\mu$ is finite and contains $\alpha$. The intersection property is evidently fulfilled and it remains to show that $V_{\alpha}$ is a normal neighborhood of $A_{\alpha}$. We take a locally finite partition of unity $\left\{\psi_{\lambda}\right\}$ on $X$ such that the sets $\left.\left.\psi_{\lambda}^{-1}\right] 0,1\right]$ have diameter at most $1 / 2$, and denote by $\mu(\lambda) \subseteq M$ the finite set of indices $\beta$ such that there is $x \in X$ with $\psi_{\lambda}(x)>0$ and $d\left(x, A_{\beta}\right)<1 / 2$. Let $\vartheta_{\lambda}: X \rightarrow I$ be an Urysohn function with $\vartheta_{\lambda} \equiv 1$ on $A_{\alpha}$, $\vartheta_{\lambda}(x)>0 \Rightarrow d\left(x, A_{\alpha}\right)<1 / 2$ and, if $\alpha \in \mu(\lambda), \vartheta_{\lambda} \equiv 0$ on $X \backslash V_{\alpha}^{\mu(\lambda)}$. We set

$$
f:=\sum_{\lambda} \psi_{\lambda} \vartheta_{\lambda}: X \rightarrow I \text {. }
$$

Then $f$ is an Urysohn function with $f \equiv 1$ on $A_{\alpha}$ and $f \equiv 0$ on $X \backslash V_{r}$.

Proposition 3. In the situation of Proposition 2 there exists a locally finite family of functions $\left\{\psi_{\alpha}\right\}$ on $X$ with $\left.\left.A_{\alpha} \subseteq \psi_{\alpha}^{-1}\right] 0,1\right] \subseteq V_{\alpha}$ and $\sum_{\alpha \alpha} \psi_{\alpha}=1$ on $\bigcup \mathscr{A}$. In particular: If $\mathscr{A}$ covers $X$ then $\left\{V_{\alpha} \mid \alpha \in M\right\}$ is a normal covering of $X$. 
Proof. We take a locally finite partition of unity $\left\{\vartheta_{\lambda}\right\}$ on $X$, such that for each $\lambda$ the set $\mu(\lambda)$ of indices $\alpha$ with $\left.\left.A_{\alpha} \cap \vartheta_{\lambda}^{-1}\right] 0,1\right] \neq \varnothing$ is finite; moreover we choose Urysohn functions $f_{\alpha}$ with $f_{\alpha} \equiv 1$ on $A_{\alpha}$ and $f_{\alpha} \equiv 0$ on $X \backslash V_{\alpha}$. Then the definition

$$
\psi_{\alpha}^{\prime}:=f_{\alpha} \sum\left\{\vartheta_{\lambda} \mid \alpha \in \mu(\lambda)\right\}
$$

leads to a locally finite family of functions with $\left.\left.\psi_{\alpha}^{\prime-1}\right] 0,1\right] \subseteq V_{\alpha}$ and $\psi_{\alpha}^{\prime} \equiv 1$ on $A_{\alpha}$. Finally we set $\Psi:=\max \left(1, \sum_{\alpha} \psi_{\alpha}^{\prime}\right)$ and $\psi_{\alpha}:=\psi_{\alpha}^{\prime} / \Psi$ to get the functions we are looking for.

Corollary 1. (a) Every admissible covering has a normal open enlargement of the same nerve.

(b) Every admissible family in an arbitrary topological space has a locally finite open enlargement.

By a Theorem of Katětov [10] every locally finite collection of subsets of a collectionwise normal, countably paracompact space has a locally finite open enlargement. Therefore Corollary 1.b raises the question whether in the range of validity of the Katětov Theorem our notion of admissible families coincides with locally finite ones. The following proposition gives a positive answer:

Proposition 4. In a collectionwise normal, countably paracompact space $X$ a family of subsets $\mathscr{A}=\left\{A_{\alpha} \mid \alpha \in M\right\}$ is admissible if and only if it is closed and locally finite.

Proof. We only have to show sufficiency, and since in a collectionwise normal space every closed subset is normally embedded only condition (ix) is in question. We take a locally finite collection of open subsets $\left\{V_{\alpha} \mid \alpha \in M\right\}$ with $A_{\alpha} \subseteq V_{\alpha}$ and choose Urysohn functions $\psi_{\alpha}$ with $\psi_{\alpha} \equiv 1$ on $A_{\alpha}$ and $\psi_{\alpha} \equiv 0$ on $X \backslash V_{\alpha}$. This gives rise to a continuous pseudometric $d$ on $X$ defined by $d(x, y):=\sum_{\alpha}\left|\psi_{\alpha}(x)-\psi_{\alpha}(y)\right|$; let $\widetilde{X}$ be the metrizable quotient space obtained from $X$ by identifying points of distance 0 . The functions $\psi_{\alpha}$ determine continuous maps $\varphi_{\alpha}: \widetilde{X} \rightarrow \mathbb{R}$ by passing to the quotient, and their sum $\Phi:=\sum_{\alpha} \varphi_{\alpha}: \widetilde{X} \rightarrow \mathbb{R}$ is continuous and finite too. The collection $\left\{\varphi_{\alpha}\right\}$ need not be locally finite on $\tilde{X}$, but it is point finite, i.e. for every $x \in \tilde{X}$ the set $M(x):=\left\{\alpha \in M \mid \varphi_{\alpha}(x)>0\right\} \subseteq M$ is finite, possibly empty. The set $W_{x}:=\left\{y \in \widetilde{X} \mid \Phi(y)<1+\sum_{\alpha \in M(x)} \varphi_{\alpha}(y)\right\}$ is an open neighborhood of $x$. Let us consider $y \in W_{x}$ and $\beta \in M \backslash M(x)$; we have $\varphi_{\beta}(y) \leq \Phi(y)-\sum_{\alpha \in M(x)} \varphi(y)<1$ and hence $y$ cannot belong to the image of $A_{\beta}$ in $\widetilde{X}$. This means that every $W_{x}$ meets the image of $A_{\beta}$ for at most finitely many indices $\beta \in M$. The sets $W_{x}$ form an open covering $\mathscr{W}$ of $\tilde{X}$, and its inverse image in $X$ may serve as $\mathscr{U}$ in condition (ix).

Now we are ready to show that the elements of an admissible covering determine the strong shape of the whole space. Therefore we need a characterization of strong shape equivalences:

Lemma 3. (a) Let $B$ be a closed subspace of a topological space $Y$, such that the inclusion map $i: B \hookrightarrow Y$ is a cofibration. Then $i$ is a strong shape equivalence if 
and only if it is an SSDR-map, i.e. if for any Hurewicz fibration $E \rightarrow P$ between ANR-spaces $E$ and $P$ the following commutative square admits a filler $Y \rightarrow E$ preserving commutativity:

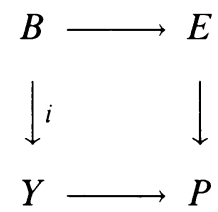

(b) If $B$ is a normally embedded subspace of a space $Y$ and if for every normal neighborhood $W$ of $B$ in $Y$ there is a deformation $D: Y \times I \rightarrow Y$ relative $B$ with $D_{0}=$ id and $D_{1}(Y) \subseteq W$, then the inclusion map $B \hookrightarrow Y$ is a strong shape equivalence.

Proof. (a) It follows from conditions (i) and (ii) that $i$ is a strong shape equivalence if and only if every map from $B$ into an ANR-space can be extended over $Y$ and if any two such extensions are homotopic relative $B$. By [4, Theorem 1.2] this is equivalent to the lifting property in (a). Statement (b) follows from [9, Corollary 2.5].

Lemma 4. For any admissible covering $\mathscr{A}=\left\{A_{\alpha} \mid \alpha \in M\right\}$ of a topological space $X$ the inclusion map $X^{\mathscr{4}} \hookrightarrow X \overline{\times} \widetilde{N}$ is a strong shape equivalence.

Proof. We consider a normal neighborhood $W$ of $X^{\mathscr{A}}$ in $X \overline{\times} \tilde{N}$ and are going to construct a deformation $D: X \overline{\times} \widetilde{N} \times I \rightarrow X \overline{\times} \widetilde{N}$ relative to $X^{\mathscr{A}}$ with $D_{0}=$ id and $D_{1}(X \overline{\times} \tilde{N}) \subseteq W$. Let $g: X \overline{\times} \tilde{N} \rightarrow I$ be an Urysohn function, which vanishes outside of $W$ and is identically 1 on $X^{\mathscr{A}}$. For any simplex $\sigma$ of $\mathscr{N}(\mathscr{A})$ we define $g_{\sigma}: X \rightarrow I$ by $g_{\sigma}(x):=\inf \{g(x, t)|t \in| \sigma \mid\}$. Then $g_{\sigma} \equiv 1$ on $A_{\sigma}$ and therefore $\left.\left.U_{\sigma}:=g_{\sigma}^{-1}\right] 0,1\right]$ is a normal neighborhood of $A_{\sigma}$ such that $U_{\sigma} \times|\sigma| \subseteq W$. Now Proposition 2 provides us with a family of normal neighborhoods $V_{\alpha}$ of $A_{\alpha}$ for $\alpha \in M$, such that $\bigcap\left\{V_{\alpha} \mid \alpha \in \mu\right\}$ is contained in $U_{\mu}$, if $\mu$ is a simplex of $\mathscr{N}(\mathscr{A})$, and else it is empty. For each index $\alpha \in M$ we choose an Urysohn function $f_{\alpha}: X \rightarrow I$ with $f_{\alpha} \equiv 1$ on $A_{\alpha}$ and $f_{\alpha} \equiv 0$ outside $V_{\alpha}$. Furthermore we apply Proposition 3 to get a locally finite partition of unity $\left\{\varphi_{\alpha} \mid \alpha \in M\right\}$ on $X$ with $\left.\left.\varphi_{\alpha}^{-1}\right] 0,1\right] \subseteq V_{\alpha}$ for each $\alpha$.

We are going to construct a locally finite partition of unity $\left\{\psi_{\beta \alpha} \mid \alpha \in M\right\}$ with some additional properties to a fixed index $\beta \in M$. We set

$$
\begin{aligned}
\psi_{\beta \beta} & :=\min \left(1, f_{\beta}+2 \varphi_{\beta}\right), \\
\psi_{\beta \alpha} & := \begin{cases}\frac{1-\psi_{\beta \beta}}{1-\varphi_{\beta}} \varphi_{\alpha}, & \varphi_{\beta} \leq 1 / 2, \alpha \neq \beta, \\
0, & \varphi_{\beta} \geq 1 / 2, \alpha \neq \beta .\end{cases}
\end{aligned}
$$

We observe $\psi_{\beta \beta} \equiv 1$ on $A_{\beta}$ and $\left.\left.\psi_{\beta \alpha}^{-1}\right] 0,1\right] \subseteq V_{\alpha}$ for every $\alpha \in M$. This leads to a map $\Psi_{\beta}: X \rightarrow \widetilde{N}$ defined by $\Psi_{\beta}(x):=\sum_{\alpha \in M} \psi_{\beta \alpha}(x) p_{\alpha}$, where $p_{\alpha}$ denotes the vertex corresponding to the index $\alpha$. On $A_{\beta}$ the function $\Psi_{\beta}$ takes the constant value $p_{\beta}$; and for any index $\alpha$ we have $\Psi_{\beta}^{-1}\left(\operatorname{St} p_{\alpha}\right) \subseteq V_{\alpha}$. 
Now we can define our deformation by

$$
D\left(x, \sum_{\beta} s_{\beta} p_{\beta}, t\right):=\left(x, \sum_{\beta} s_{\beta}\left\{(1-t) p_{\beta}+t \Psi_{\beta}(x)\right\}\right) .
$$

All required properties of $D$ are obvious with the exception of the inclusion relation $D_{1}(X \overline{\times} \tilde{N}) \subseteq W$. Therefore let us consider a point $\left(x, \sum_{\beta} s_{\beta} p_{\beta}\right) \in$ $X \overline{\times} \tilde{N}$ and set $(x, q):=D_{1}\left(x, \sum_{\beta} s_{\beta} p_{\beta}\right)=\left(x, \sum_{\beta} s_{\beta} \Psi_{\beta}(x)\right)$. If $\mu \subseteq M$ is the finite collection of indices $\alpha$ with $q \in \mathrm{St} p_{\alpha}$, then we have $x \in V_{\alpha}$ for every such $\alpha$. This means that $\mu$ is a simplex of $\mathcal{N}(\mathscr{A})$ and $x \in U_{\mu}$, and so we have $(x, q) \in U_{\mu} \times|\mu| \subseteq W$. Since by Proposition $1 X^{\mathscr{A}}$ is normally embedded in $X \overline{\times} \widetilde{N}$ Lemma 3.b tells us that the inclusion map is a strong shape equivalence.

The canonical projection $X \overline{\times} \tilde{N} \rightarrow X$ induces a map $p^{\mathscr{A}}: X^{\mathscr{A}} \rightarrow X$ by restriction, and since $\widetilde{N}$ is contractible Lemma 4 implies

Theorem 1. The natural projection map $p^{\mathscr{A}}: X^{\mathscr{A}} \rightarrow X$ is a strong shape equivalence.

Theorem 1 should be compared with a Theorem of G. Segal saying that $p^{\mathscr{A}}: X^{\mathscr{A}} \rightarrow X$ is a homotopy equivalence if $\mathscr{A}$ is a normal open covering [16, Proposition 4.1]. Our Theorem 2 is modelled after a Theorem of T. tom Dieck valid in the same situation as the G. Segal Theorem:

Theorem 2. Suppose we are given two spaces $X$ and $Y$, admissible coverings $\mathscr{A}=\left\{A_{\alpha} \mid \alpha \in M\right\}$ of $X$ and $\mathscr{B}=\left\{B_{\alpha} \mid \alpha \in M\right\}$ of $Y$ with the same index set $M$ and a map $f: X \rightarrow Y$ with $f\left(A_{\alpha}\right) \subseteq B_{\alpha}$ for all $\alpha \in M$. If for every nonvoid subset $\mu \subseteq M$ the restricted mapping $f_{\mu}: A_{\mu} \rightarrow B_{\mu}$ is a strong shape equivalence, then $f$ is a strong shape equivalence.

Proof. At first we treat the special case where every $f_{\mu}: A_{\mu} \rightarrow B_{\mu}$ is an SSDRmap. By Theorem 1 it suffices to show that the obvious map $F: X^{\mathscr{A}} \rightarrow Y^{\mathscr{B}}$ is an SSDR-map, and to this end we consider a fibration $\pi: E \rightarrow P$ between ANR-spaces and two maps $\varphi: X^{\mathscr{A}} \rightarrow E, \psi: Y^{\mathscr{B}} \rightarrow P$ with $\pi \varphi=\psi F$. By [4, Corollary 1.6] $A_{\mu} \times|\mu| \cup B_{\mu} \times \partial|\mu| \rightarrow B_{\mu} \times|\mu|$ is an SSDR-map for every $\mu$, and therefore we can construct a family of maps $\omega_{\mu}: B_{\mu} \times|\mu| \rightarrow E$ by induction on the number of elements of $\mu$, such that $\omega_{\mu}\left(f_{\mu} \times\right.$ id $)$ equals the restriction of $\varphi, \pi \omega_{\mu}$ equals the restriction of $\psi$ and such that for $\nu \supseteq \mu$ the maps $\omega_{\nu}$ and $\omega_{\mu}$ coincide on $B_{\nu} \times|\mu|$. The collection of all these maps determine a map $\omega: Y^{\mathscr{B}} \rightarrow E$ with $\omega F=\varphi$ and $\pi \omega=\psi$, and $F$ is shown to be an SSDR-map.

To deal with the general case we cover the mapping cylinder $Z=Y \cup_{f} X \times I$ by the sets $C_{\alpha}:=B_{\alpha} \cup A_{\alpha} \times I$; it is readily checked that these sets form an admissible covering of $Z$. The inclusion of the bottom of the mapping cylinder of $f_{\mu}: A_{\mu} \rightarrow B_{\mu}$ is by assumption a strong shape equivalence for every finite, nonvoid $\mu \subseteq M$, and by Lemma 3.a it is an SSDR-map. From the special case treated before we learn that the inclusion map $X \hookrightarrow Z$ is a strong shape equivalence, and therefore $f: X \rightarrow Y$ is a strong shape equivalence too. 
Let $f: X \rightarrow Y$ be a map between paracompact spaces and $\mathscr{B}$ a locally finite, closed covering of $Y$, such that for each finite collection of elements $B_{1}, \ldots B_{n} \in \mathscr{B}$ the restricted mapping $f: f^{-1}\left(\bigcap_{k=1}^{n} B_{k}\right) \rightarrow \bigcap_{k=1}^{n} B_{k}$ is a strong shape equivalence. By [7, Theorem 2.9] $f$ is a strong shape equivalence. To see that our Theorem 2 extends this result we simply have to observe that the coverings $\mathscr{B}$ and $\mathscr{A}:=\left\{f^{-1}(B) \mid B \in \mathscr{B}\right\}$ are admissible. The reason that two coverings enter our Theorem in contrast to the simpler formulation of the Dydak-Nowak Theorem is, that continuous inverse images of admissible coverings need not be admissible, because the inverse image of a normally embedded subspace is not necessarily normally embedded. For a counterexample one considers a space $X$, which is not collectionwise normal, and a closed subspace $A$ of $X$, which is not normally embedded. If $p: X \rightarrow X / A$ denotes the quotient map, then $B:=p(A) \subset X / A$ as a one point space is normally embedded in $X / A$, although $A=p^{-1}(B)$ is not normally embedded in $X$.

\section{APPLICATIONS}

Theorem 3. If a space $X$ has an admissible covering $\mathscr{A}$, such that every finite intersection of elements of $\mathscr{A}$ has trivial shape, then $X$ is of trivial shape.

Remark 3. It has been shown by Koyama, Mardešić and Watanabe [11] that a space has trivial strong shape if and only if it has trivial ordinary shape. We emphasize that by a space $Y$ of trivial shape we mean one having the shape of a one point space, so that in particular $Y$ cannot be empty.

Proof. It follows readily from conditions (i) and (ii) that a space $Y$ has trivial shape if and only if every map from $Y$ into an ANR-space is homotopic to a constant map, and if for every $n>0$ every map from $Y \times S^{n}$ into an ANRspace has an extension over $Y \times E^{n+1}$. By induction over the cells of $N(\mathscr{A})$ we see that $X^{\mathscr{}}$ has this property, therefore by Theorem $1 X$ has trivial shape.

Example 1. The above theorem is clearly false, if "strong shape" is replaced by "homotopy type", as the reader can see from an example taken from Spanier's book [18, p. 56, exercise A5]: Let $A:=\left\{(x, y) \in \mathbb{R}^{2} \mid 0 \leq y \leq 1, x=\right.$ $0,1 / n$ or $y=1,0 \leq x \leq 1\}$ be the comb space, and let $B$ be the space obtained from $A$ by reflection at the origin. Then $A, B$ and $A \cap B$ are contractible compact metric spaces, but $A \cup B$ is not contractible.

The skeletal filtration on the polyhedron $N(\mathscr{A})$ gives rise to a filtration on $X^{\mathscr{A}}$ : We denote by $N_{k}$ the $k$-skeleton of $N(\mathscr{A})$, set $X_{k}^{\mathscr{A}}:=X^{\mathscr{A}} \cap\left(X \times N_{k}\right)$ and observe that $X_{k+1}^{\mathscr{A}}$ is obtained from $X_{k}^{\mathscr{\otimes}}$ by attaching the spaces $A_{\sigma} \times|\sigma|$ via the inclusion map $A_{\sigma} \times \partial|\sigma| \hookrightarrow X_{k}^{\mathscr{}}$ with $\sigma$ running through the $k+1$ simplexes of $\mathscr{N}(\mathscr{A})$. The main advantage of this filtration is the cofibration property of the inclusion maps $X_{k}^{\mathscr{A}} \hookrightarrow X_{m}^{\mathscr{A}}$.

Theorem 4. If a space $X$ has an admissible covering $\mathscr{A}$ of finite dimension $n$, such that every finite intersection of elements of $\mathscr{A}$ has shape dimension at most $m$, then the shape dimension of $X$ does not exceed $n+m$.

For the proof we need the following lemma:

Lemma 5. For every space $X$ we have $\operatorname{sd}\left(X \times S^{n}\right) \leq n+\operatorname{sd} X$. 
Proof. The case $n=0$ is obvious, so suppose the lemma holds for $n-1$. If $H_{+}$and $H_{-}$denote the upper respectively lower hemisphere of $S^{n}$, then we have $H_{+} \cap H_{-}=S^{n-1}$ and hence $\operatorname{sd}\left(\left(X \times H_{+}\right) \cap\left(X \times H_{-}\right)\right) \leq n-1+\operatorname{sd} X$. Now [9, Theorem 1.2] implies $\operatorname{sd}\left(X \times S^{n}\right) \leq n+\operatorname{sd} X$.

Proof of Theorem 4. By Theorem 1 it suffices to show $\operatorname{sd} X^{\mathscr{A}} \leq m+n$. To this end we consider a map $f: X^{\mathscr{A}} \rightarrow P$ into a polyhedron $P$ and have to construct a map $g: X^{\mathscr{A}} \rightarrow P$ homotopic to $f$, whose image is contained in $P_{m+n}$, the $m+n$-skeleton of $P$. Applying [9, Lemma 2.8.b] to the inclusions $A_{\sigma} \times \partial|\sigma| \subseteq A_{\sigma} \times|\sigma|$ we get a sequence of maps $g_{k}: X^{\mathscr{A}} \rightarrow P$ with $g_{k}\left(X_{k}^{\mathscr{A}}\right) \subseteq$ $P_{m+k}$ and $g_{k+1} \simeq g_{k}$ relative $X_{k}^{\mathscr{A}}$. Then $g_{n}$ has the desired property.

Corollary 2. For every space $X$ and every locally compact polyhedron $P$ we have $\operatorname{sd}(X \times P) \leq \operatorname{sd} X+\operatorname{dim} P$.

Proof. One considers the covering of $X \times P$ consisting of the sets $X \times|\sigma|$ with $\sigma$ ranging over the simplexes of a triangulation of $P$ and applies Theorem 4.

Remark 4 . With a slightly different approach the estimate in Theorem 4 can be strengthened to $\operatorname{sd} X \leq \sup _{\sigma}\left(\operatorname{sd} A_{\sigma}+\operatorname{dim} \sigma\right)$ with $\sigma$ running over all simplexes of $\mathscr{N}(\mathscr{A})$. One has to consider the barycentric subdivision of $N(\mathscr{A})$ and uses the full subpolyhedron of $N(\mathscr{A})$ spanned by the barycenters of simplexes of dimension $\geq n-k$ as $N_{k}$ in the definition of $X_{k}^{\mathscr{A}}$. The details are left to the reader.

Lemma 6. (a) We consider two direct sequences of topological spaces $f_{m}^{n}: X_{m} \rightarrow$ $X_{n}$ and $g_{m}^{n}: Y_{m} \rightarrow Y_{n}$ and a sequence of maps $h_{n}: X_{n} \rightarrow Y_{n}$ with $g_{m}^{n} h_{m}=$ $h_{n} f_{m}^{n}$. If all the bonding maps $f_{m}^{n}$ and $g_{m}^{n}$ are cofibrations and if all $h_{n}$ are strong shape equivalences, then $\varliminf_{n} h_{n}: \varliminf_{n} X_{n} \longrightarrow Y_{n}$ is a strong shape equivalence.

(b) We consider spaces and maps as in the following diagram. If $f: X \rightarrow$ $Y$ and $g: A \rightarrow B$ are strong shape equivalences, then the map between the adjunction spaces $F: X \cup_{\varphi} A \times E^{n} \rightarrow Y \cup_{\psi} B \times E^{n}$ composed of $f$ and $g \times$ id is a strong shape equivalence.

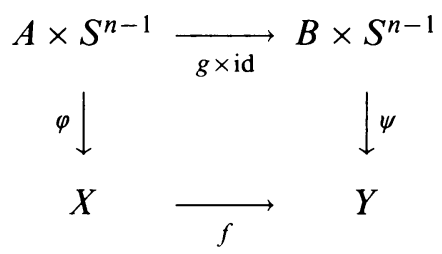

Proof. Since the constructions are functorial we may assume that the strong shape equivalences are in fact SSDR-maps, and in (b) we may furthermore assume that either $f$ or $g$ is an identity map. But then the statements follow immediately from Lemma 3.a.

Theorem 5. If a space $X$ possesses an admissible covering $\mathscr{A}$, such that each finite intersection of elements of $\mathscr{A}$ has the strong shape of a CW-space, then $X$ has the strong shape of a $C W$-space.

Proof. By Theorem 1 it suffices to show that $X^{\mathscr{A}}$ has the strong shape of a $\mathrm{CW}$-space. We will construct a sequence of $\mathrm{CW}$-spaces $Y_{k}$ and strong shape 
equivalences $F_{k}: X_{k}^{\mathscr{A}} \rightarrow Y_{k}$, such that $Y_{k+1}$ containes $Y_{k}$ as a subcomplex and $F_{k+1}$ extends $F_{k}$, then our Theorem follows from Lemma 6.a.

For each simplex $\sigma$ of $\mathscr{N}(\mathscr{A})$ we choose a $\mathrm{CW}$-complex $P_{\sigma}$ and a strong shape equivalence $f_{\sigma}: A_{\sigma} \rightarrow P_{\sigma}$. For $Y_{0}$ we can use the disjoint union of the complexes $P_{\alpha}$ with $\alpha$ ranging over the vertices of $\mathscr{N}(\mathscr{A}), F_{0}$ is obvious. Now we suppose $Y_{k}$ and $F_{k}$ and are already constructed and set $\widetilde{A}$ and $\widetilde{P}$ equal to the disjoint union of the spaces $A_{\sigma}$ respectively $P_{\sigma}$ with $\sigma$ ranging over the the $k+1$-simplexes of $\mathscr{N}(\mathscr{A})$; the strong shape equivalences $f_{\sigma}: A_{\sigma} \rightarrow P_{\sigma}$ provide us with a strong shape equivalence $g: \widetilde{A} \rightarrow \widetilde{P}$. We observe that $g \times$ id : $\widetilde{A} \times S^{n-1} \rightarrow \widetilde{P} \times S^{n-1}$ is a strong shape equivalence too and denote by $i: \widetilde{A} \times S^{n-1} \hookrightarrow X_{k}^{\mathscr{A}}$ the inclusion map. Since $Y_{k}$ has the homotopy type of an ANR-space we can find a cellular map $\psi: \widetilde{P} \times S^{n-1} \rightarrow Y_{k}$ and a homotopy $\Psi: F_{k} i \simeq \psi(g \times \mathrm{id})$.

We realize the $n$-cell in euclidean $n$-space as $E^{n}=\left\{t \in \mathbb{R}^{n} \mid\|t\| \leq 1\right\}$ and set $C^{n}:=\left\{t \in E^{n} \mid\|t\| \geq 1 / 2\right\}, S^{n-1} \subset C^{n} \subset E^{n}$. Then we take a look at the following two pushout diagrams of inclusion maps:

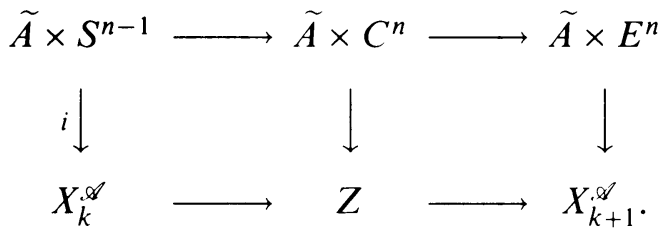

The space $Z$ is defined by the diagram; $X_{k}^{\mathscr{A}}$ is a strong deformation retract of $Z$. We define $F^{\prime}: Z \rightarrow Y_{k}$ to be equal to $F_{k}$ on $X_{k}^{\mathscr{A}}$, whereas on $\tilde{A} \times C^{n}$ we set $F^{\prime}(x, t):=\Psi(x, t /\|t\|, 2\|t\|-1) . F^{\prime}$ is a strong shape equivalence and fits commutatively into the following diagram:

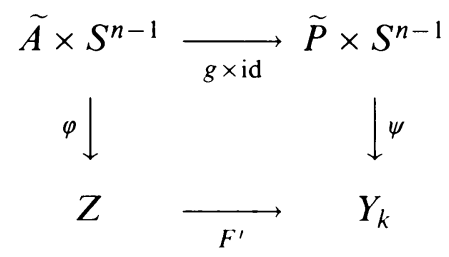

with $\varphi(x, t):=\left(x, \frac{1}{2} t\right)$. We observe $X_{k+1}^{\mathscr{A}}=Z \cup_{\varphi} \tilde{A} \times E^{n}$, set $Y_{k+1}:=$ $Y_{k} \cup_{\psi} \widetilde{P} \times E^{n}$ and apply Lemma 6 .b to get a strong shape equivalence $F_{k+1}$ : $X_{k+1}^{\mathscr{A}} \rightarrow Y_{k+1}$ extending $F_{k}: X^{\mathscr{A}} \rightarrow Y_{k}$.

Example 1 shows that Theorem 5 does not hold for homotopy type instead of strong shape, because the spaces $A, B$ and $A \cap B$ have the homotopy type of CW-spaces, but $X$ has not.

The Leray spectral sequence constructed in [16, pp. 110-111] can be set up for admissible coverings and for every homology ${ }^{1}$ or cohomology functor factoring over the strong shape category, but for infinite dimensional coverings it converges only in a very weak sense. The reader's attention should be drawn

\footnotetext{
${ }^{1}$ Of course the Leray spectral sequence for strong homology must not be confused with the Bousfield-Kan spectral sequence. The latter one is of Adams type, the former one is not.
} 
to the fact that even in the case of finite dimensional coverings Segal's proof is hardly applicable beyond the realm of paracompact spaces, because his strong excision axiom (iii) and the exactness axiom (i) together are very restrictive. For Čech cohomology or strong homology one of the two axioms (which one, depends on the definition) is known to hold in general, but the other one only for normally embedded subspaces. To solve this problem we use our filtration $X_{k}^{\mathscr{A}}$; since the inclusion maps $X_{k}^{\mathscr{A}} \hookrightarrow X_{m}^{\mathscr{A}}$ are always cofibrations and a fortiori normal embeddings the homology or cohomology sequences of these pairs are exact. Hence the definition

$$
H(p, q):=\sum_{n \in \mathbb{Z}} h_{n}\left(X_{-p}^{\mathscr{A}}, X_{-q}^{\mathscr{A}}\right) \quad \text { for }-\infty \leq p \leq q \leq \infty,
$$

where $h_{*}$ is a possibly generalized homology functor and $X_{-\infty}^{\mathscr{A}}=\varnothing, X_{\infty}^{\mathscr{A}}=$ $X^{\mathscr{A}}$, provides us with an $H(p, q)$-system as in [3, p. 335, example 3]. Conditions (SP.1)-(SP.4) from [3, p. 334] are satisfied, but (SP.5) need not hold. This forces us to restrict our attention to finite dimensional coverings, but the calculation of the $E_{* *}^{1}$-terms requires further restriction to finite coverings:

$$
\begin{aligned}
E_{p q}^{1} & =E_{1}^{-p,-q}=h_{p+q}\left(X_{p}^{\mathscr{A}}, X_{p-1}^{\mathscr{A}}\right) \\
& \approx \tilde{h}_{p+q}\left(X_{p}^{\mathscr{A}} / X_{p-1}^{\mathscr{A}}\right) \\
& \approx \tilde{h}_{p+q}\left(\bigvee_{\sigma} A_{\sigma}^{+} \wedge S^{p}\right) \\
& \approx h_{q}\left(\coprod_{\sigma} A_{\sigma}\right)
\end{aligned}
$$

where $\sigma$ runs over the set of $p$-simplexes of $\mathscr{N}(\mathscr{A})$. By [14, Theorem 3] we cannot prove $h_{q}\left(\bigsqcup_{\sigma} A_{\sigma}\right) \approx \bigoplus_{\sigma} h_{q}\left(A_{\sigma}\right)$ unless the index set is finite. If this assumption is satisfied, then we learn in [3, Chapter $15, \S 7]$ how to construct a spectral sequence converging to $h_{p+q}\left(X^{\mathscr{A}}\right)$, and if $h_{*}$ factors over the strong shape category and $\mathscr{A}$ is admissible, then this is isomorphic to $h_{p+q}(X)$.

We summarize:

Theorem 6. For every homology functor $h_{*}$ factoring over the strong shape category and every finite, admissible covering $\mathscr{A}$ of a space $X$ indexed by a linearly ordered set there is a spectral sequence $E_{* *}^{r}$ of bidegree $(-r, r-1)$ with $E_{p q}^{1}=\bigoplus_{\sigma} h_{q}\left(A_{\sigma}\right)$ (the sum being taken over all p-simplexes of $\mathscr{N}(\mathscr{A})$ ) converging to $h_{p+q}(X)$. The differential $d: E_{p q}^{1} \rightarrow E_{p-1, q}^{1}$ maps an element $\zeta \in h_{q}\left(A_{\sigma}\right)$ to $\sum_{i=0}^{p}(-1)^{i} h_{q}\left(j_{i}\right)(\zeta)$, where $j_{i}: A_{\sigma} \hookrightarrow A_{\partial_{i} \sigma}$ is the inclusion map.

We observe that the ordering on the index set is necessary to define an orientation of $S^{p}$ in (4) and to give a well-defined meaning to the $i$ th face $\partial_{i} \sigma$ of a simplex $\sigma$ in Theorem 6 .

The whole scheme may be set up for cohomology as well; one gets an Adams type spectral sequence. Čech cohomology is infinitely additive, so that in this case we may consider infinite coverings. In order to deal with convergence we 
either have to restrict our attention to finite dimensional coverings or to use $\lim ^{1}$ short exact sequences. The details are left to the reader.

\section{REFERENCES}

1. Richard A. Alo and Harvey L. Shapiro, Normal toplogical spaces, Cambridge Tracts in Math., vol. 65, Cambridge Univ. Press, 1974.

2. J. M. Boardman, Conditionally convergent spectral sequences, Preprint Baltimore, 1981.

3. H. Cartan and S. Eilenberg, Homological algebra, Mathematical Series, vol. 19, Princeton Univ. Press, 1956.

4. F. W. Cathey, Strong shape theory, Shape Theory and Geom. Top. Proc., Dubrovnik 1981 (S. Mardešić and J. Segal, eds.), Lecture Notes in Math., vol. 870, Springer, 1981, pp. 215238.

5. F. W. Cathey and J. Segal, Strong shape theory and resolutions, Topology Appl. 15 (1983), 119-130.

6. T. tom Dieck, Partitions of unity in homotopy theory, Compositio Math. 23 (1971), 159-167.

7. J. Dydak and S. Nowak, Strong shape for topological spaces, Trans. Amer. Math. Soc. 323 (1991), 765-796.

8. R. Engelking, General topology, 2nd ed., Heldermann, Berlin, 1989.

9. B. Günther, Properties of normal embeddings concerning strong shape theory, I, Tsukuba J. Math. 15 (1991), 261-274.

10. M. Katětov, Extensions of locally finite coverings, Colloq. Math. 6 (1958), 145-151.

11. A. Koyama, S. Mardešić, and T. Watanabe, Spaces which admit AR-resolutions, Proc. Amer. Math. Soc. 102 (1988), 749-752.

12. L. L. Krajewski, On expanding locally finite collections, Canad. J. Math. 23 (1971), 58-68.

13. Ju. T. Lisica and S. Mardešić, Coherent prohomotopy and strong shape theory, Glasnik Mat. 19(39) (1984), 335-399.

14. S. Mardešić and A. V. Prasolov, Strong homology is not additive, Trans. Amer. Math. Soc. 307 (1988), 725-744.

15. S. Mardešić and J. Segal, Shape theory, Mathematical Library, vol. 26, North-Holland, 1982.

16. G. Segal, Classifying spaces and spectral sequences, Publ. Math. Inst. Hautes Etudes. Sci. 34 (1968), 105-112.

17. J. C. Smith and L. L. Krajewski, Expandability and collectionwise normality, Trans. Amer. Math. Soc. 160 (1971), 437-451.

18. E. H. Spanier, Algebraic topology, McGraw-Hill, 1966.

Fachbereich Mathematik, Johann Wolfgang Goethe-Universität, Robert-MaYerStrasse 6-10, 6000 Frankfurt, GermanY

E-mail address: guenther@math.uni-frankfurt.de 